\title{
SOME INVESTIGATIONS ON THE ANISOTROPY OF THE CHEMICAL ETCHING OF (hk0) AND (hhl) SILICON PLATES IN A NaOH 35\% SOLUTION. PART I: 2D ETCHING SHAPES
}

\author{
C. A. HODEBOURG ${ }^{a}$ and C. R. TELLIER ${ }^{b, *}$ \\ ${ }^{a}$ Laboratoire de Chronométrie Electronique et Piezoélectricité, \\ Ecole Nationale Supérieure de Mécanique et des Microtechniques, \\ 26 chemin de l'Epitaphe, 25030 Besançon cédex France; \\ ${ }^{\mathrm{b}}$ Institut des Microtechniques de Franche-Comté, Avenue de l'Observatoire, \\ 25030 Besançon cédex France
}

(Received 1 February 2001; In final form 19 March 2001)

In this paper a study of the anisotropic dissolution of (hk0) and (hhl) silicon plates in a $\mathrm{NaOH} 35 \%$ solution is undertaken. Effects of orientation on firstly, the geometrical features of etched surfaces and secondly, on the cross-sectional shape of starting circular plates are systematically investigated. Conclusions of practical interest on the roughness of etched (hk0) and (hhl) planes are drawn. 2D etching shapes are then analysed in terms of the tensorial model for the anisotropic dissolution and of dissolution criteria. Finally a comparative analysis of results related on the one hand, to 2D surface profiles and on the other, to out-of-roundness profiles is made. This comparison shows that shapes observed for profilometry traces agree with theoretical shapes as derived when we use the resemblance in shapes between out-of-roundness profiles and polar diagrams of the dissolution slowness.

Keywords: Anisotropic etching; Silicon; NaoH etchant

*Corresponding author. Tel.: 03-81-40-28-30, Fax: 03-81-88-57-14, e-mail: ctellier@ ens $2 \mathrm{~m} . \mathrm{fr}$ 


\section{INTRODUCTION}

In the last decade a great lot of works [1-9] was devoted to the study of various etching baths dedicated to the micromachining of silicon crystal. To obtain with reproducibility mechanical microstructures of definite shape and size it is advantageous to use highly anisotropic etchants. So it is usual to work with etching baths which possess the particularity to dissolve very slowly $\{111\}$ silicon planes [1-7]. EDP and $\mathrm{KOH}$ based solutions [1-5] are currently used but in the past few years new etching baths such as TMAH [5-7], Cesium hydroxide [8] and $\mathrm{NaOH}+$ IPA solutions [9] were investigated. This paper continues on this line and reports some results on the anisotropy of a pure $\mathrm{NaOH} 35 \%$ solution. The paper is divided into two parts, the first part is mainly concerned with $2 \mathrm{D}$ etching shapes such as surface profiles and out-of-roundness profiles corresponding to cross-sections of etched thick silicon plates whereas the second part deals with 3D structures micromachined in various (hk0) and (hhl) silicon planes. A practical point of view is adopted in the two parts of this paper and emphasis is placed on technical problems encountered in wet micromachining processes such as the degradation of etched surfaces, the identification of facets limiting microstructures, the importance of concave or convex undercutting and the formation of terraces on edges bounding membranes and mesa.

\section{EXPERIMENTAL DETAILS}

To study the changes in starting 2D shapes induced by chemical etching differently oriented thick circular silicon plates $(10 \mathrm{~mm}$ in diameter, initial thickness $d_{o}=1.5 \mathrm{~mm}$ ) were cut in a silicon ingot. The angles of cut as defined (Fig. 1) in the IEEE Standard on Piezoelectricity [10] are, for the various $\{\mathrm{hk} 0\}$ and $\{\mathrm{hhl}\}$ plates, listed in Table I. The two sides and the contour of plates were firstly mechanically lapped and then polished (initial arithmetic roughness $\mathrm{Ra} \approx 0.01 \mu \mathrm{m}$ ). All plates were immersed in a $35 \%$ in weight $\mathrm{NaOH}$ solution maintained at a constant $\left( \pm 0.5^{\circ}\right)$ temperature of $60^{\circ} \mathrm{C}$. After 


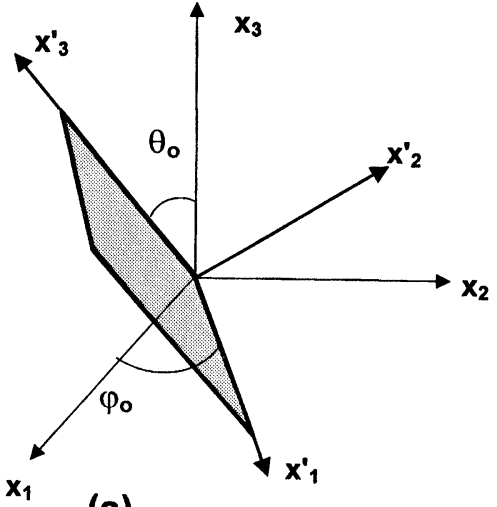

(a)

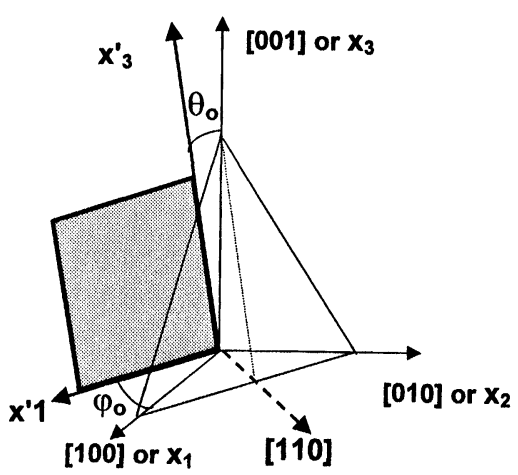

(b)

FIGURE 1 Definition of the angles of cut for a doubly rotated plate (a) and for an (hhl) plane (b).

TABLE I Values for the angle of cut $\varphi_{\mathrm{o}}$ of $\{\mathrm{hk} 0\}$ plates and for the second angle of cut $\theta_{\mathrm{o}}$ of $\{\mathrm{hhl}\}$ plates. Only Miller indices smaller than 10 are indicated

\begin{tabular}{|c|c|c|c|}
\hline \multicolumn{2}{|c|}{$(h \bar{k} 0)$ planes } & \multicolumn{2}{|c|}{ (hhl) planes } \\
\hline$h k 0$ & $\varphi_{\mathrm{o}}\left(^{\circ}\right)$ & hhl & $\theta_{0}\left({ }^{\circ}\right)$ \\
\hline 010 & 0 & 661 & 6,7 \\
\hline- & 5 & 441 & 10 \\
\hline 610 & 10 & 331 & 13,2 \\
\hline 410 & 14 & 221 & 19,47 \\
\hline 730 & 23 & 111 & 35,23 \\
\hline 740 & 30 & 112 & 57,7 \\
\hline 320 & 34 & 113 & 64,7 \\
\hline 430 & 37 & 114 & 70,5 \\
\hline 980 & 42 & 115 & 74,21 \\
\hline 110 & 45 & 118 & 80 \\
\hline
\end{tabular}

isothermal etchings of duration $\Delta t=1 \mathrm{~h}$ following investigations were performed:

(1) Decrements in thickness $\Delta \mathrm{d}$ were measured using a Palmer instrument.

(2) Modifications of the contour shape of starting circular plates were investigated using a Talyrond analyser which furnishes an out-of-roundness profile superimposed to the least mean square circle. 
(3) Geometrical features of etched surfaces were observed by Scanning Electron Microscopy. In addition a computer based mechanical profilometer gives 3D topographies of surfaces and 2D profilometry traces made along two specific directions. For the $\{h k 0\}$ plates the two directions are the rotated $x_{1}^{\prime}$ axis (Fig. 1) and the [001] direction. For the $\{\mathrm{hhl}\}$ plates traces lie parallel to the primed $x_{3}^{\prime}$ axis and to the $\langle 110\rangle$ direction. Roughness parameters such as the centre-line average $\mathrm{Ra}$, the root-mean-square roughness $\mathrm{Rq}$ and the peak-to-valley height $\mathrm{Rmax}$ were evaluated.

\section{EXPERIMENTAL RESULTS}

\subsection{The Etch Rate}

For the silicon crystal which belongs to the class $\mathrm{m} 3 \mathrm{~m}$ the dissolution rates for the two faces of a $\{\mathrm{hk} 0\}$ or of a $\{\mathrm{hhl}\}$ plate are similar. Consequently the dissolution rate $\mathbf{R}$ for a face is simply given by:

$$
\mathrm{R}\left(\varphi_{\mathrm{o}}, \theta_{\mathrm{o}}\right)=\frac{1}{2} \Delta \mathrm{d}
$$

where $\Delta \mathrm{d}$ is the decrement in thickness.

Variations in the etch rate $R$ with the angle of cut $\varphi_{\mathrm{o}}$ ( $\{\mathrm{hk} 0\}$ plates) or with the angle $\theta_{\mathrm{o}}(\{\mathrm{hhl}\}$ plates) are drawn in Figure 2. Turning attention to $\{\mathrm{hk} 0\}$ plates we observe that $\{100\}$ plates $\left(\varphi_{\mathrm{o}}=0^{\circ}\right)$ dissolve more slowly than $\{110\}$ plates $\left(\varphi_{0}=45^{\circ}\right)$. Moreover the maximum of the etch rate $R\left(\varphi_{0}\right)$ occurs for $\varphi_{o} \approx 26^{\circ}$ that is to say for $\{120\}$ planes. So for $\{\mathrm{hk0}\}$ planes the anisotropy is crudely similar to those observed for EDP [1, 2, 3], TMAH [6] and $\mathrm{KOH}[1,3]$ etchings. The variations in the etch rate $\mathrm{R}\left(\varphi_{\mathrm{o}}=-45^{\circ}, \theta_{\mathrm{o}}\right)$ with the angle of cut $\theta_{\mathrm{o}}$ show as expected a marked minimum for the (111) plane and a minor minimum for the (001) plane $\left(\theta_{o}=90^{\circ}\right)$. Moreover for (hhl) planes, the etch rate presents two maxima: a major maximum for $\theta_{\mathrm{o}} \approx 65^{\circ}$ and a slightly less accentuated minimum for $\theta_{\mathrm{o}}=0^{\circ}$ (i.e., for a $\{110\}$ plane). In terms of the dissolution slowness $[11,12]$ we thus conclude that certainly the dissolution slowness surface exhibits in the one hand, valleys associated with planes close to $\{113\}$ planes and in the second hand, two types of protuberances. Elongated protuberances 

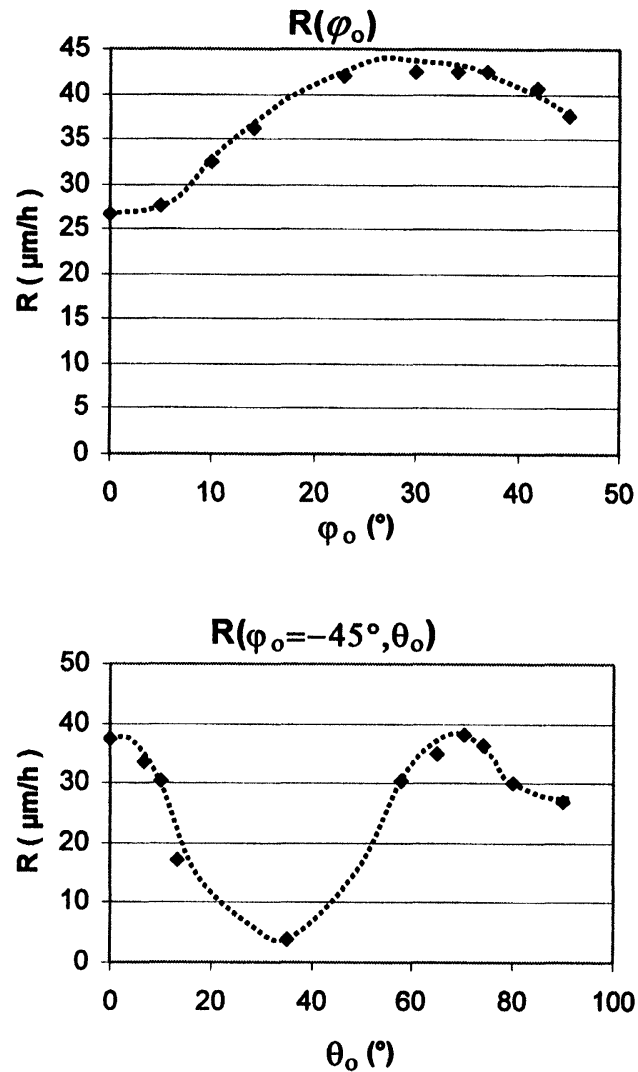

FIGURE 2 Variations in etch rates $R\left(\varphi_{\mathrm{o}}\right)\left(\{\mathrm{hk} 0\}\right.$ plates) and $\mathrm{R}\left(\varphi_{\mathrm{o}}=-45^{\circ}, \theta_{\mathrm{o}}\right)(\{\mathrm{hhl}\}$ plates) with the angles of cut $\varphi_{\mathrm{o}}$ and $\theta_{\mathrm{o}}$ respectively.

correspond to $\{111\}$ planes whereas small peaks appear for $\{100\}$ planes. Comparison of changes in $\mathrm{R}\left(\varphi_{\mathrm{o}}\right)$ and in $\mathrm{R}\left(\varphi_{\mathrm{o}}=-45^{\circ}, \theta_{\mathrm{o}}\right)$ for cuts in the vicinity of the (110) plane indicates that $\{110\}$ planes do not correspond to a minor peak in the dissolution slowness as observed for various etching baths such $\mathrm{KOH} \mathrm{35 \%} \mathrm{[3]} \mathrm{and} \mathrm{TMAH}$ $25 \%[6]$.

\subsection{The Topography of Etched Surfaces}

SEM images of $\{\mathrm{hk} 0\}$ and $\{\mathrm{hhl}\}$ etched surfaces are displayed in Figures 3 to 6 . Etched surfaces are covered by dissolution figures 

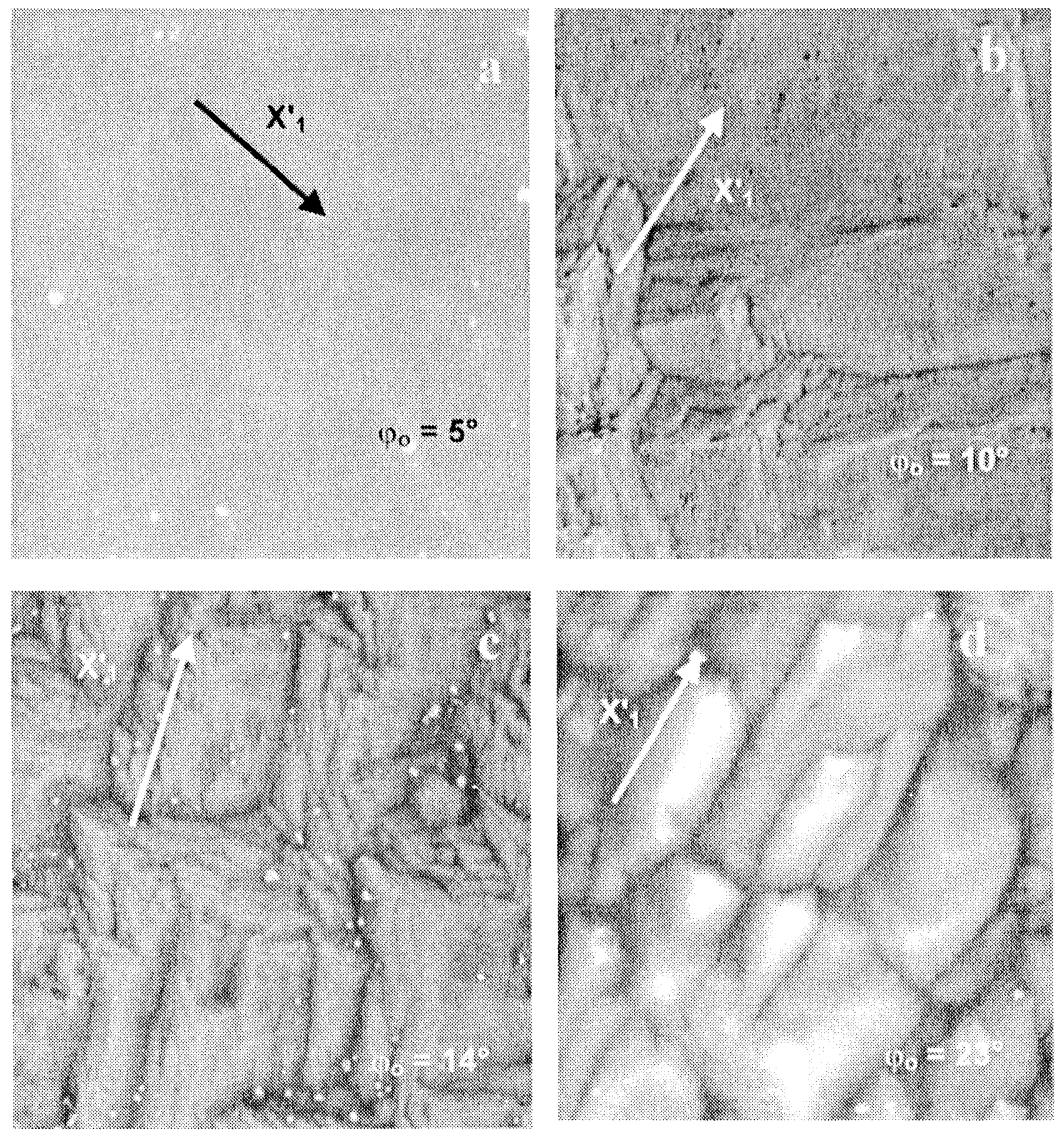

FIGURE 3 SEM images of etched $\{$ hk 0$\}$ plates, a, b, c and $d$ are for $\varphi_{0}=5^{\circ}, \varphi_{0}=10^{\circ}$, $\varphi_{\mathrm{o}}=14^{\circ}$ and $\varphi_{\mathrm{o}}=23^{\circ}$ respectively. Arrows indicate the direction of the rotated axis $\mathrm{x}_{1}^{\prime}$.

characteristic of the cut. For all the $\{\mathrm{hk} 0\}$ plates investigated here the dissolution figures are aligned along the rotated axis $x_{1}^{\prime}$, but the more elongated dissolution figures develop on plates close to $\{100\}$ plates or to $\{120\}$ plates. When the angle of cut is in the range $34^{\circ}-45^{\circ}$ the dissolution figures seem to be partly limited by $\{111\}$ facets and partly composed of small hillocks. Moreover contrary to other etchings the dissolution of $\{110\}$ and $\{980\}$ plates in a $\mathrm{NaOH} 35 \%$ solution causes the formation of somewhat pyramidal figures. Typical features of etched $\{\mathrm{hk} 0\}$ plates are listed in Table II. 

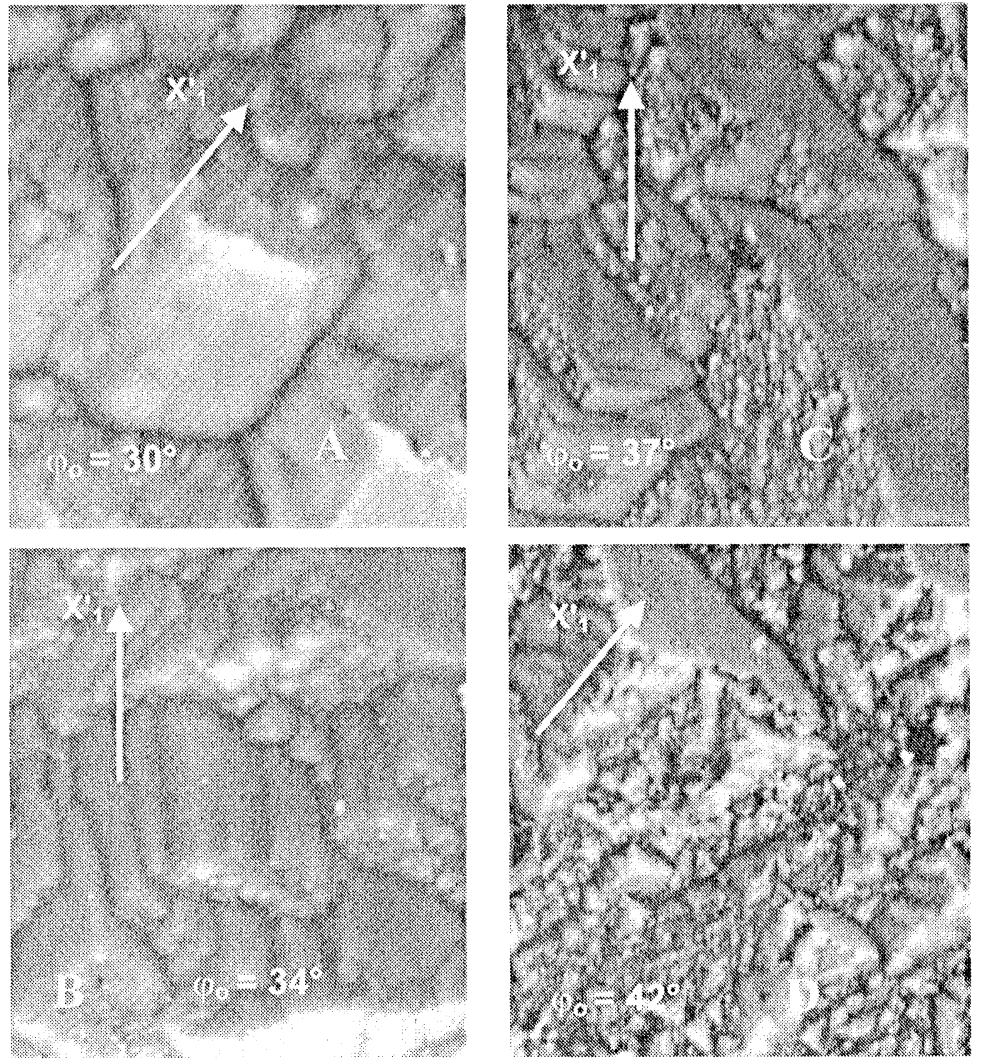

FIGURE 4 SEM images of etched $\{$ hk 0$\}$ plates, A, B, C and D are for $\varphi_{0}=30^{\circ}$, $\varphi_{\mathrm{o}}=34^{\circ}, \varphi_{\mathrm{o}}=37^{\circ}$ and $\varphi_{\mathrm{o}}=42^{\circ}$ respectively. Arrows indicate the direction of the rotated axis $\mathbf{x}_{1}^{\prime}$.

Let us now turn attention to (hhl) plates and recall that when the angle of cut $\theta_{\mathrm{o}}$ increases from $0^{\circ}\left((110)\right.$ plane, Fig. 6D) to $90^{\circ}((001)$ plane, Fig. 5a) the (hhl) plane passes through the (111) plane. Examination of SEM images of Figures 5 and 6 reveals that:

(1) Etching causes dissolution figures to appear whose shape changes progressively with the angle of cut $\theta_{\mathbf{o}}$. For convenience Table II summarises this evolution in shape.

(2) Dissolution figures which develop on (hhl) plates seem to be more ((551) plane) and less ((112) plane) elongated in a direction $\mathrm{x}_{\mathbf{3}}^{\prime}$ which is perpendicular to the direction $\langle 110\rangle$. 
(3) Most of dissolution figures (see Figs. 5b, 5c, 5d for example) appear to be symmetrical with respect to the cross-sectional $\{110\}$ plane.

(4) (hhl) plates in the vicinity of the (001) plane $\left(90^{\circ} \geq \theta_{0}>62^{\circ}\right)$ show the less degraded surfaces. In particular smooth elongated bumps are formed on etched (114) and (113) surfaces. When $\theta_{0}$ decreases from $55^{\circ}$ to $10^{\circ}\{111\}$ terraces with steps partly covered by small "pyramidal" hillocks are generated yielding surfaces of poor quality.

\subsection{Profilometry Traces}

Since the dissolution figures are aligned along specific directions which for (hk0) and (hhl) plates correspond to the rotated $\mathrm{x}_{1}^{\prime}$ axis and $\mathrm{x}_{\mathbf{3}}^{\prime}$
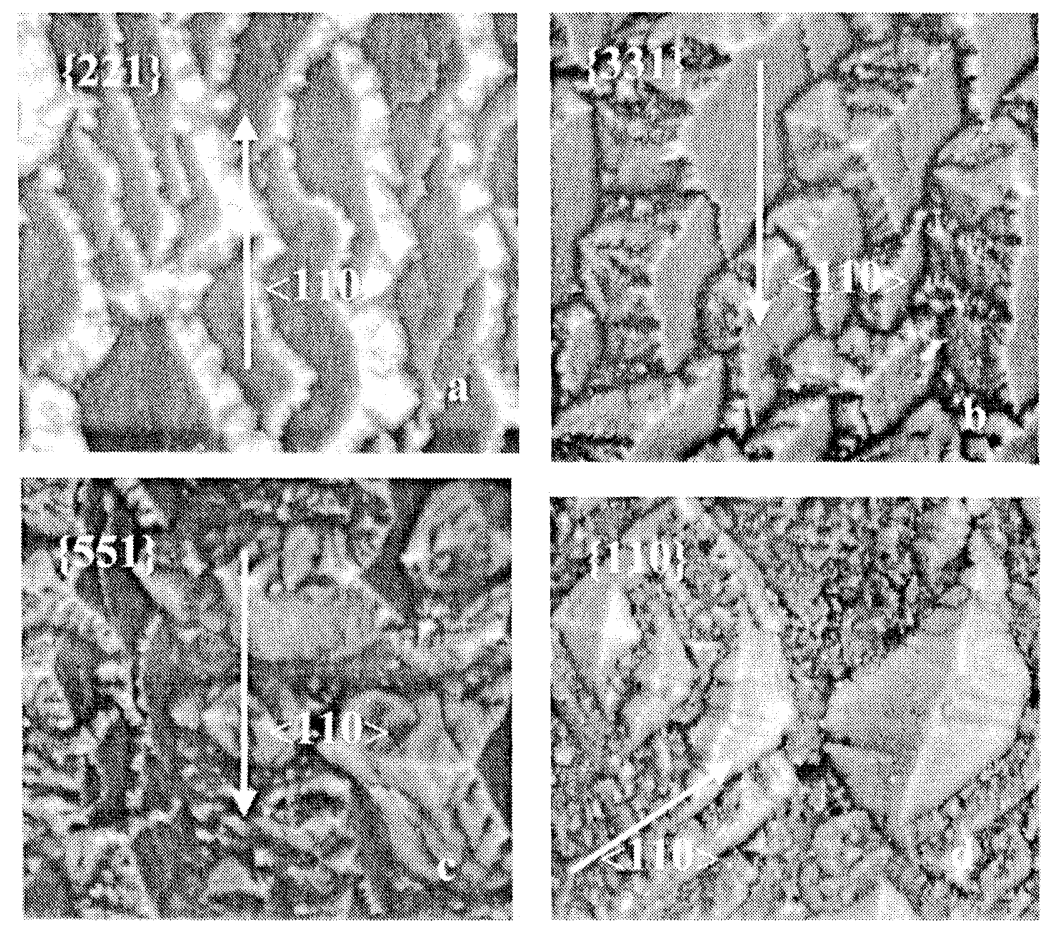

FIGURE 5 SEM images of etched (hhl) plates, a, b, c and d are for (221), (331), (551) and (110) planes respectively. Arrows indicate the direction of the rotated axis $x_{1}^{\prime}(\langle 110\rangle$ direction). 

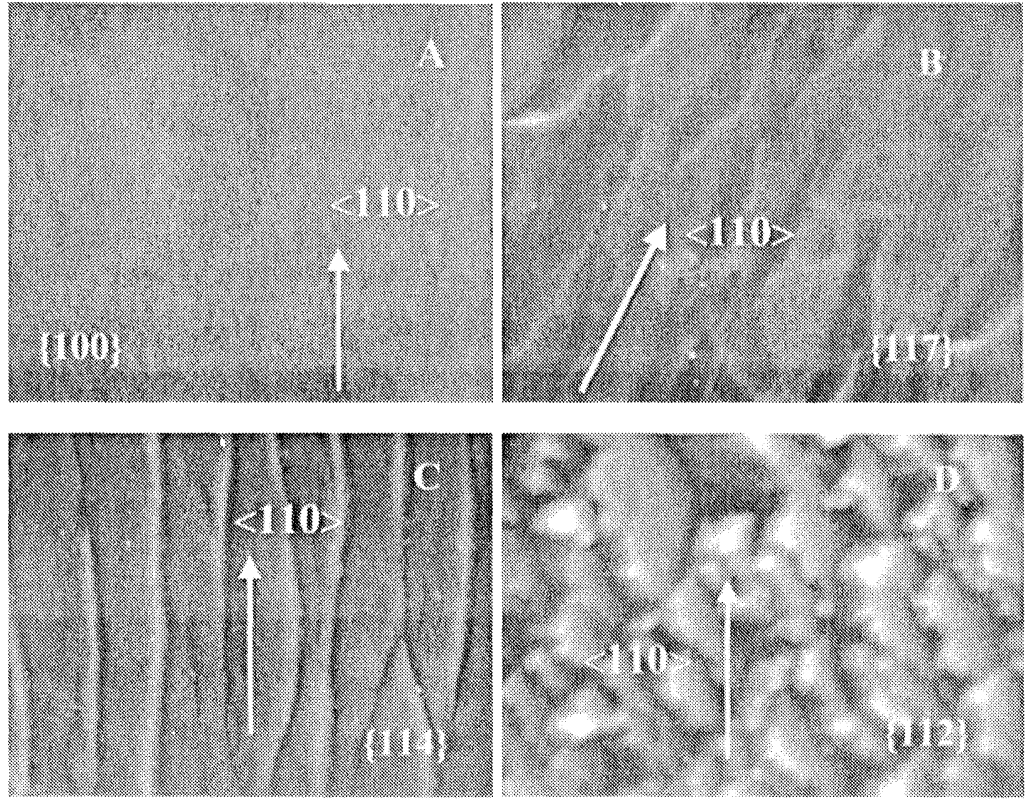

FIGURE 6 SEM images of etched (hhl) plates, A, B, C and D are for (100), (117), (114) and (112) planes respectively. Arrows indicate the direction of the rotated axis $\mathrm{x}_{1}^{\prime}$ $(\langle 110\rangle$ direction).

TABLE II Geometrical features of dissolution figures formed on (hk0) and (hhl) planes

\begin{tabular}{llcl}
\hline Planes & \multicolumn{1}{c}{ Geometrical features } & Planes & \multicolumn{1}{c}{ Geometrical features } \\
\hline$(010)$ & Flat pits & $(551)$ & Sharp hillocks \\
$(610)$ & Somewhat concave terraces & $(441)$ & Sharp hillocks \\
$(410)$ & Terraces & $(331)$ & Terraced hillocks \\
$(730)$ & Elongated bumpy hillocks & $(221)$ & Terraces \\
$(740)$ & Bumpy hillocks & $(112)$ & Terraced surface \\
$(320)$ & Terraced hillocks & $(113)$ & Very flats elongated hillocks \\
$(430)$ & Terraced hillocks & $(114)$ & Very flats elongated hillocks \\
$(980)$ & Some pyramidal hillocks present & $(115)$ & Somewhat convex-concave pits \\
$(110)$ & Pyramidal hillocks present & $(117)$ & Elongated pits \\
\hline
\end{tabular}

respectively it is very interesting to study changes in shape of profilometry traces made along these directions and along the directions which are perpendicular to directions of alignment (i.e., $\langle 110\rangle$ direction for (hhl) planes and [001] direction for (hk0) plates). 

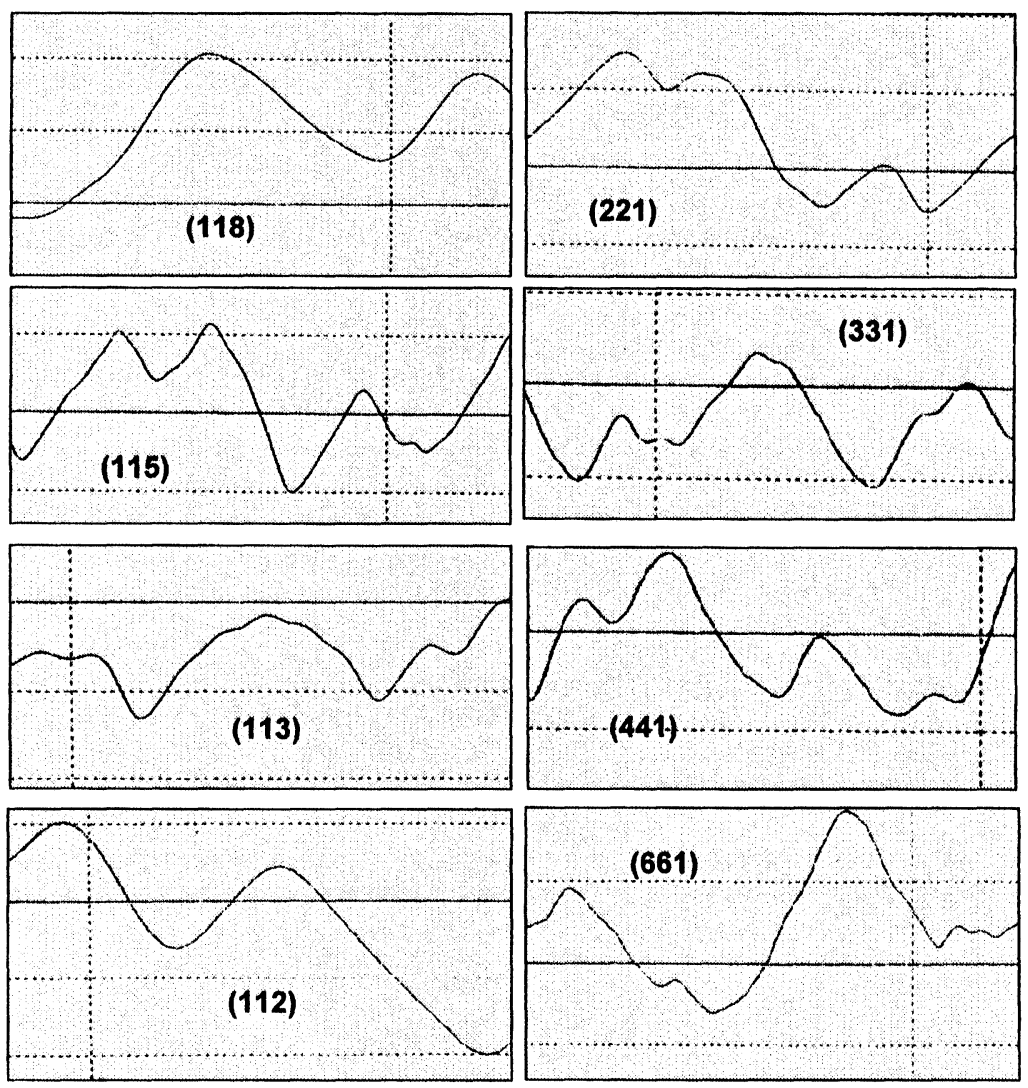

FIGURE $7 \mathrm{x}_{3}^{\prime}$ profilometry traces made on various (hhl) plates as indicated on diagrams.

Figures 7 to 10 illustrate the changes in shapes of these traces with the angles of cut. Several features emerge from these figures:

(1) The evolution of the shape for $x_{3}^{\prime}$ traces appears to be progressive. As the plate is rotated from (110) plate to the (001) plate the shape is progressively modified from convex for the (110) plate to concave for the (001) plate. For (hhl) plates corresponding to intermediate orientations $\left(6^{\circ}<\theta_{\mathrm{o}}<35^{\circ}\right.$ and $\left.36^{\circ}<\theta_{\mathrm{o}}<81^{\circ}\right)$ the $\mathrm{x}_{3}^{\prime}$ trace exhibits an alternate concave-convex shape ((111) plates) and convex-concave shape ((hhl) plates). 
Let us recall that to analyse the shape of a 2D surface profile we have to consider the polar diagram of the dissolution slowness $\mathbf{L}$ $[12,13]$ lying in the cross-sectional $\{110\}$ plane which intersects the (hhl) plane along the $\mathrm{x}_{3}^{\prime}$ axis. Consequently the observed changes in shape can be easily explained if we keep in mind that when the angle of cut $\theta_{\mathrm{o}}$ varies from $0^{\circ}$ to $90^{\circ}$ the dissolution slowness vector $\mathbf{L}$ of an (hhl) plane moves along the polar diagram corresponding to the (110) plane.

(2) An unlike behaviour can be depicted (Fig. 8) for $\langle 110\rangle$ traces made on (hhl) planes. Effectively in this case various cross-sectional planes concern us so that no progressive changes in shape can occur. In fact all $\langle 110\rangle$ traces show symmetrical shapes with respect to the vertical axis. Hence these traces satisfy the mirror symmetry specific to $\{110\}$ planes since the cross-sectional section of a given (hhl) plane is necessary an other $\left(\mathrm{h}^{\prime} \mathrm{h}^{\prime} \mathrm{l}^{\prime}\right)$ plane.

(3) Turning now attention to traces made on (hk0) plates it clearly appears that $\mathrm{x}_{1}^{\prime}$ traces follow also a progressive evolution of shape according to the scheme (Fig. 9):

$$
\begin{aligned}
& \text { Concave }(\{100\} \text { planes }) \rightarrow \text { concave }- \text { convex } \rightarrow \text { convex } \\
& (\{120\} \text { planes }) \rightarrow \text { convex }- \text { concave } \\
& (\{320\} \text { to }\{980\} \text { planes })
\end{aligned}
$$

As in remark (1) we attribute this evolution to the fact that for (hk0) plates the final etching shapes of the $2 \mathrm{D} \mathrm{x}_{1}^{\prime}$ traces are all determined from the (001) polar diagram of the dissolution slowness [2].

(4) The behaviour for [001] traces made on (hk0) plates (Fig. 10) is close to that observed in (2) for $\langle 110\rangle$ traces on (hhl) planes with firstly, not any changes in shape with the angle of cut $\varphi_{\mathrm{o}}$ and secondly, with profiles that verify the mirror symmetry associated now with the (001) plane.

\subsection{Surface Roughness}

Several works $[14,15]$ have given evidence that four-pole piezoresistive elements diffused on (hhl) planes may be conveniently used to measure 

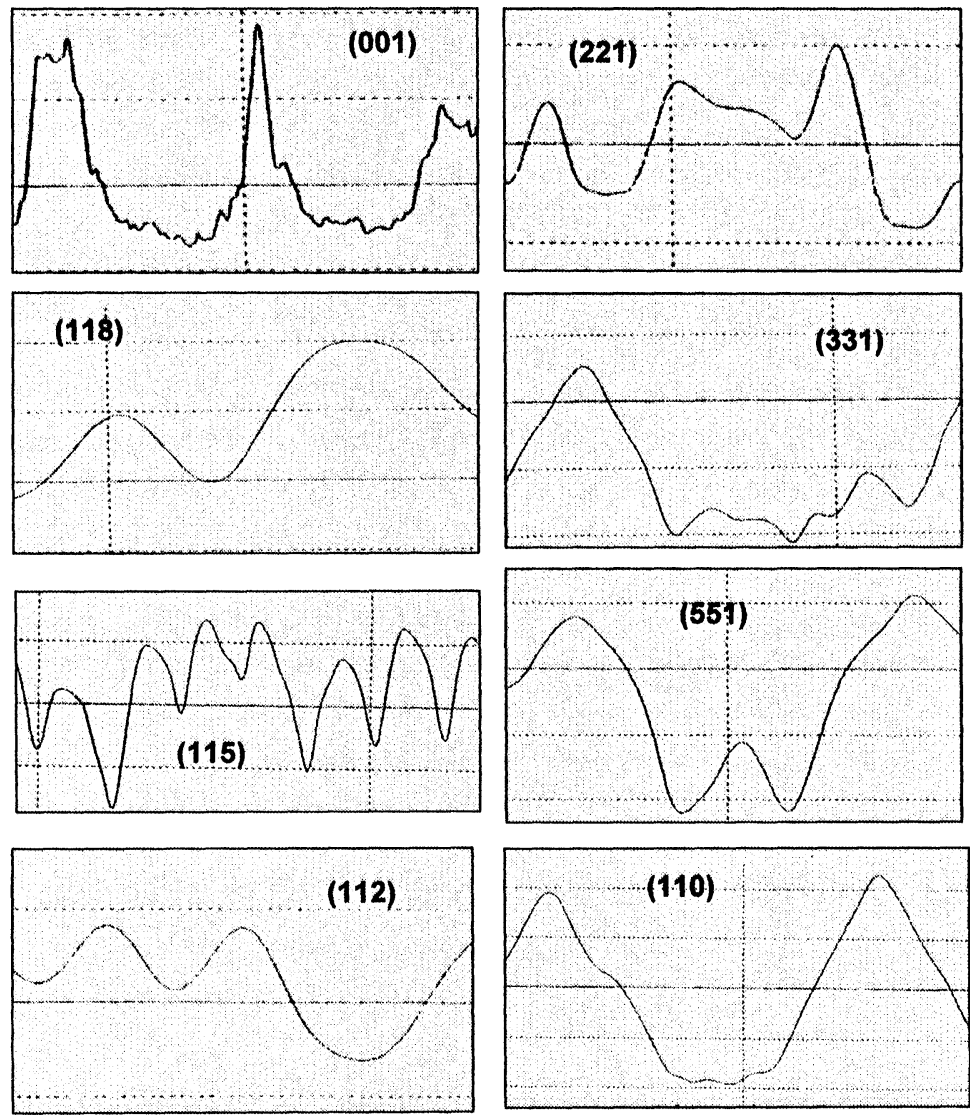

FIGURE $8\langle 110\rangle$ profilometry traces made on various (hhl) plates as indicated on diagrams.

three in-plane stresses (uniaxial stresses $T_{11}^{\prime}$ and $T_{33}^{\prime}$, shear stress $T_{13}^{\prime}$ ). For such applications mechanical devices must be micromachined in (hhl) planes. Consequently it is interesting to study the roughness of etched surfaces which can significantly limit the metrological performances of mechanical sensors.

For this purpose roughness parameters $\mathrm{Ra}, \mathrm{Rq}$, and $\mathrm{Rmax}$ were evaluated from $x_{3}^{\prime}$ and $\langle 110\rangle$ traces made on (hhl) planes. Variations in the roughness parameters $\mathrm{Ra}$ and $\mathrm{Rq}$ (in dotted lines) as a function of 

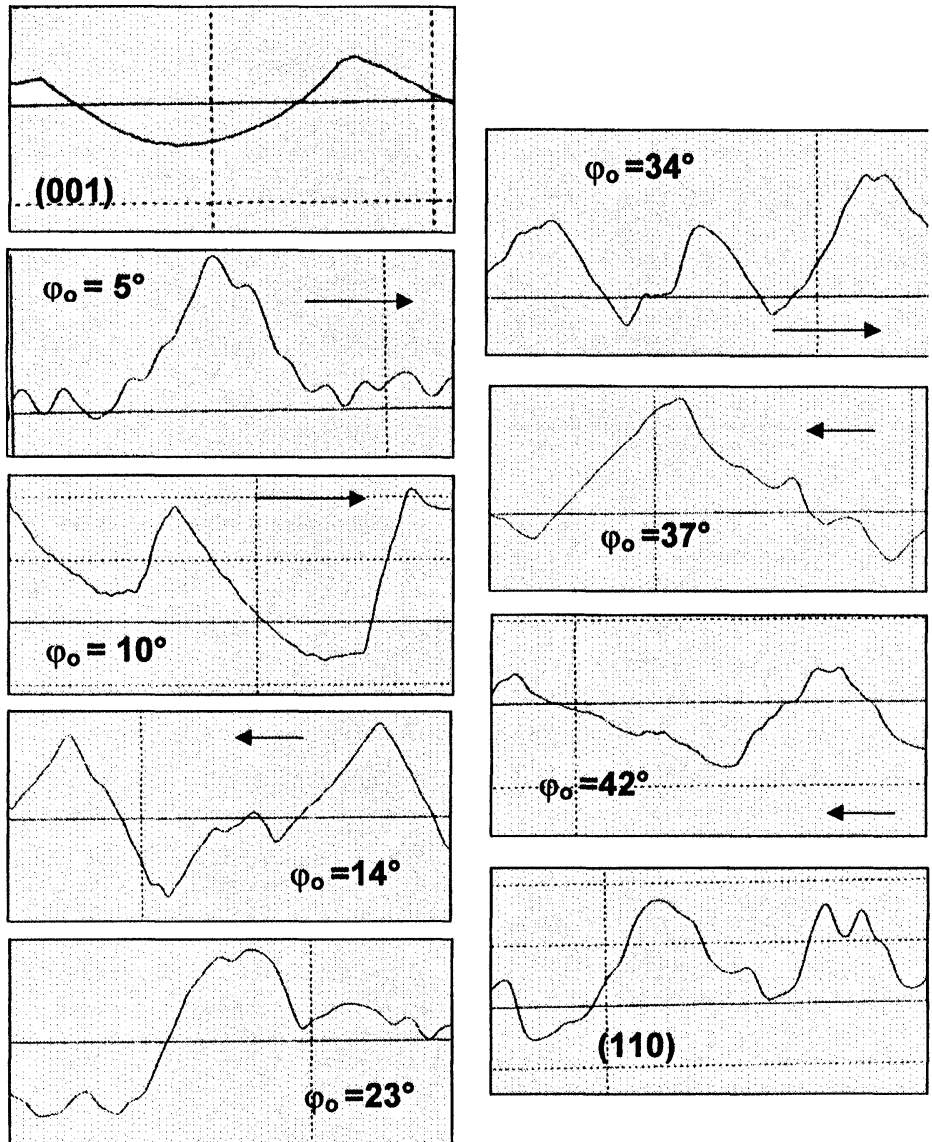

FIGURE $9 \mathrm{x}_{1}^{\prime}$ profilometry traces made on various (hk0) plates. Values of the angle of cut $\varphi_{\mathrm{o}}$ are indicated on diagrams. For convenience arrows show the $\mathrm{x}_{1}^{\prime}$ direction with respect to the direction of the trace.

the angle of cut $\theta_{\mathrm{o}}$ are drawn in Figure 11. Note that these plots which are fitted on an average behaviour give evidence for a marked degradation of etched surfaces when (hhl) planes approach the (110) plane. We effectively observe in the one hand, that roughness parameters decrease when the (hhl) plane is rotated toward the (001) plane and in the other hand, that (111) surfaces become rougher and rougher as the miller index 1 takes higher values. In particular for the 

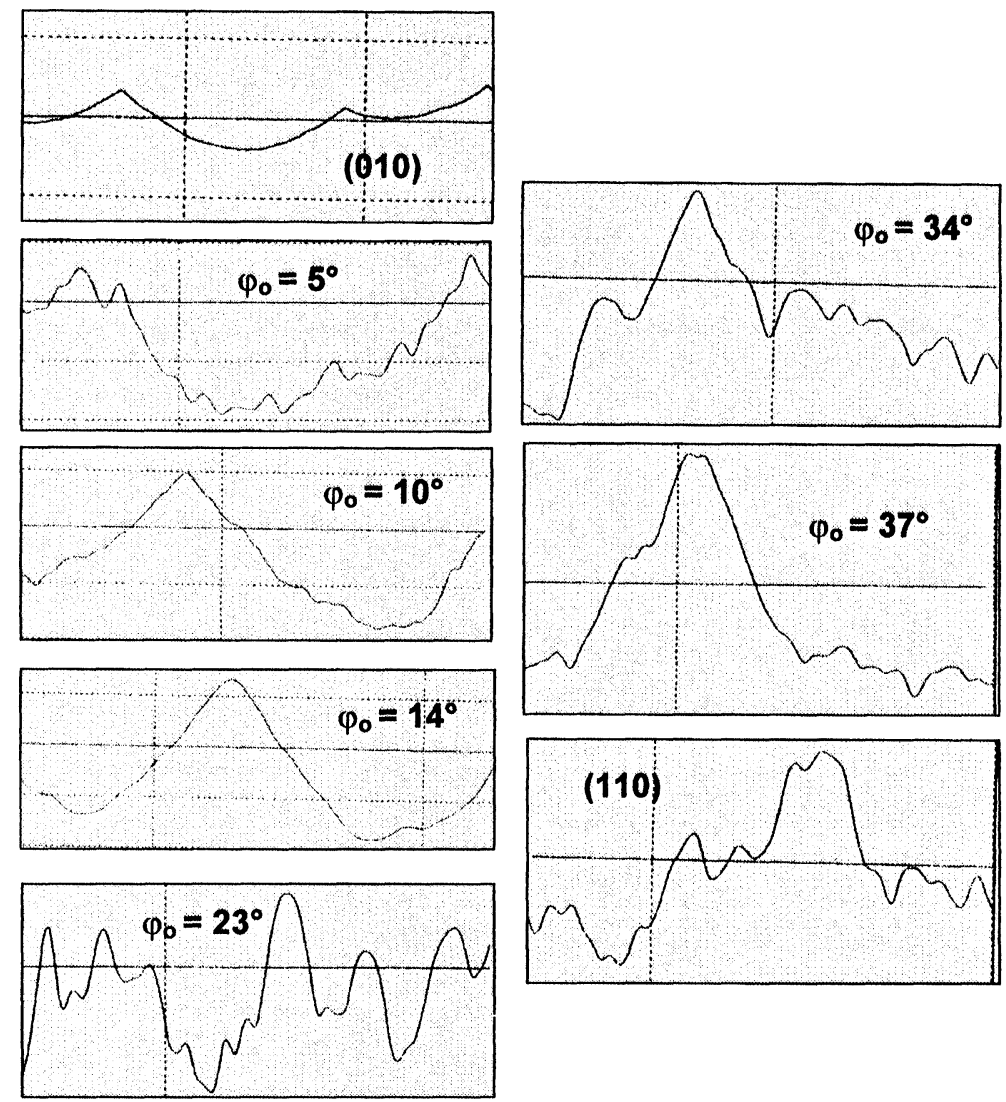

FIGURE 10 [001] profilometry traces made on various (hk0) plates. Values of the angle of cut $\varphi_{\mathrm{o}}$ are indicated on diagrams.

etched (110) surface a maximum value of $1 \mu \mathrm{m}$ is obtained for the roughness parameter Ra. For (hhl) cuts in the vicinity of the (110) plane the peak-to-valley parameter Rmax reaches values of about $5 \mu \mathrm{m}$, so these cuts must be avoided for numerous micromachining applications.

Figure 12 gives complementary results on the roughness of etched (hk0) plates. We observed that for etched $\{\mathrm{hk} 0\}$ plates the most degraded surfaces are obtained for cuts close to (320) plate for which the Rmax parameter (curve in dotted line) takes values higher than $4 \mu \mathrm{m}$. 


\section{Roughness $\{\mathrm{hhl}\}$ planes, $<110>$ axis}

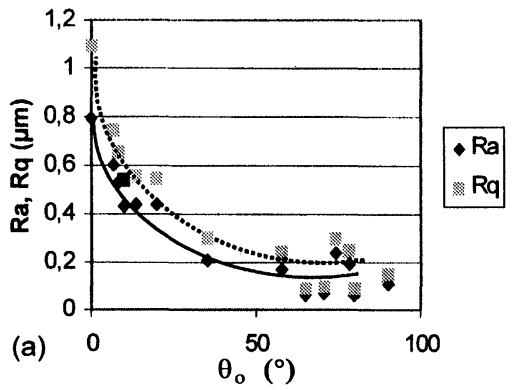

\section{Roughness, $\{$ hhl $\}$ planes, $x_{3}{ }_{3}$ axis}

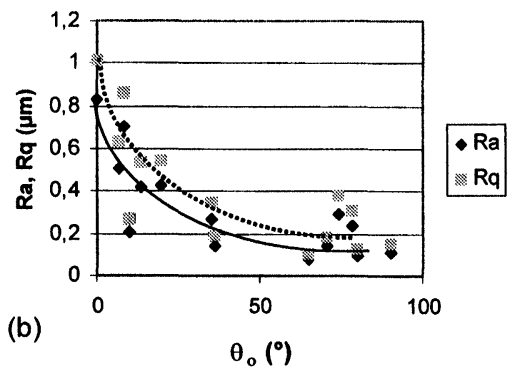

Rmax $\{h$ hl\} planes

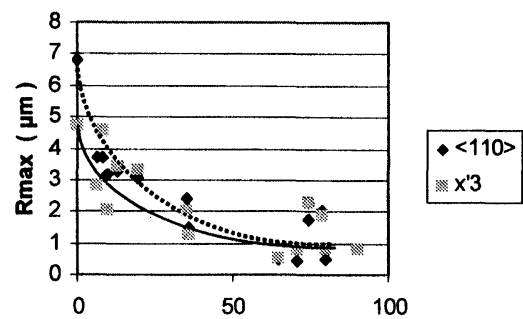

(c) $\quad \theta_{\mathrm{o}}\left({ }^{\circ}\right)$

FIGURE 11 Variations in the roughness parameters $\mathrm{Ra}$ and $\mathrm{Rq}$ as a function of the angle of cut $\theta_{\mathrm{o}}$ of $(\mathrm{hhl})$ planes. A and B are for traces made along the $\langle 110\rangle$ direction and the $\mathrm{x}_{3}^{\prime}$ axis respectively. Full lines and dotted lines are for the parameters $\mathrm{Ra}$ and $\mathrm{Rq}$ respectively. In $\mathrm{C}$ is shown the evolution of the parameter $\mathrm{Rmax}$ where here full lines and dotted lines are for the $x_{\mathbf{3}}^{\prime}$ and $\langle 110\rangle x_{\mathbf{3}}^{\prime}$ traces respectively.

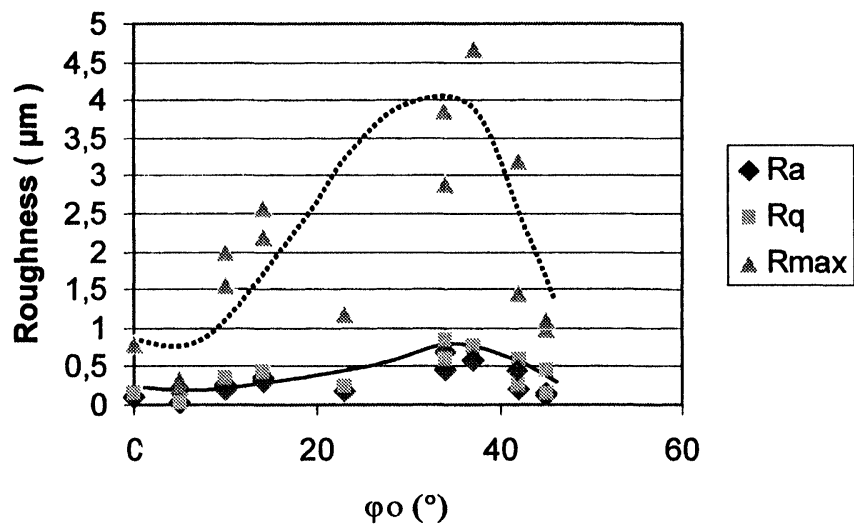

FIGURE 12 Changes in the average roughness parameters $\mathrm{Ra}, \mathrm{Rq}$ and $\mathrm{Rmax}$ with the angle of cut $\varphi_{0}$ of etched (hk0) plates. 


\section{CROSS-SECTIONAL SHAPES OF STARTING THICK CIRCULAR PLATES}

In this section we use out-of-roundness profiles to study the modification of the cross-section of starting circular plates. First consider the out-of-roundness profiles related to etched (hk0) plates (Fig. 13). The arrows on Figure 13 indicate the [001] direction, which are common to all (hk0) plates. The most significant and rapid changes (Figs. 13a to 13c) in the shape of out-of-roundness profiles are obtained when the angle of cut $\varphi_{\mathrm{o}}$ varies from $0^{\circ}\left((010)\right.$ plane) to $14^{\circ}$ ((140) plane). In contrast the out-of-roundness profiles look crudely similar (Figs. 13d to 13f) for etched (hk0) plates whose angle of cut $\varphi_{\mathrm{o}}$ is in the $\left[34^{\circ}-45^{\circ}\right]$ range. For the (110) out-of-roundness profile the four more pronounced peaks lie in the $\langle 111\rangle$ directions. Hence as observed [1-9] for other etching baths (EDP, KOH, TMAH) the \{111\} planes are certainly associated with large protuberances of the dissolution slowness surface. It is also convenient to examine
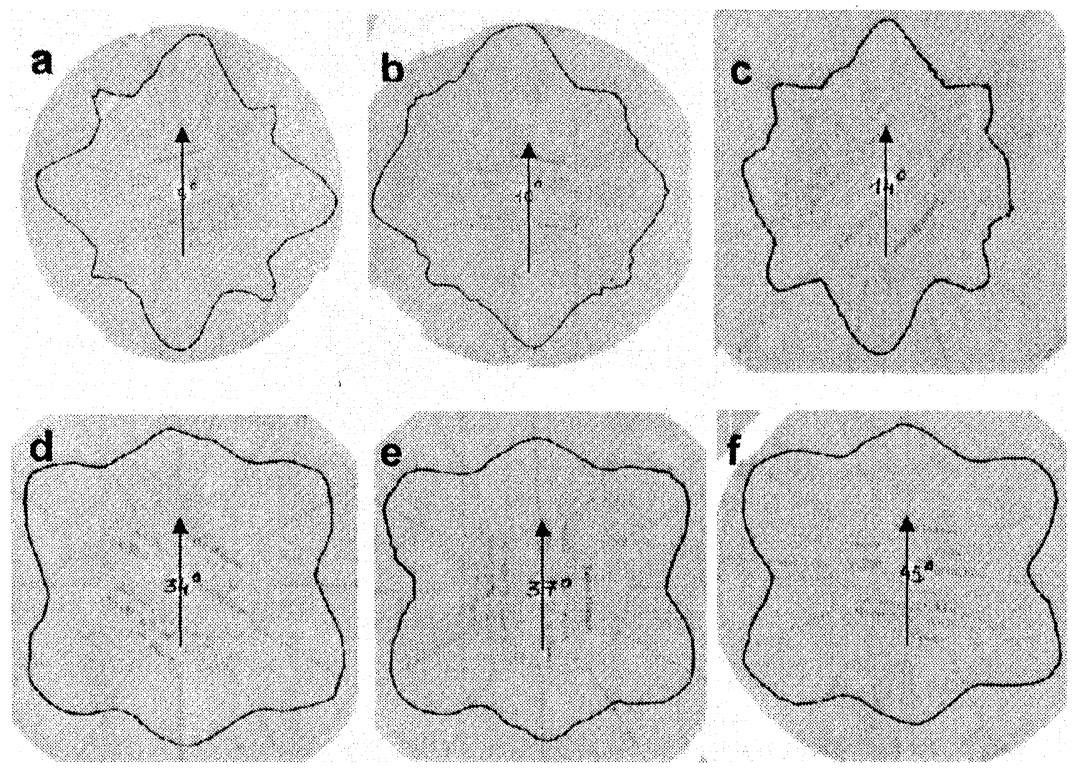

FIGURE 13 Orientation effects in the final out-of-roundness profiles related to various (hk0) sections. a, b, c, d, e and f are for $\varphi_{\mathrm{o}}=5^{\circ}, \varphi_{\mathrm{o}}=10^{\circ}, \varphi_{\mathrm{o}}=14^{\circ}, \varphi_{\mathrm{o}}=34^{\circ}, \varphi_{\mathrm{o}}=37^{\circ}$, and $\varphi_{\mathrm{o}}=45^{\circ}$ respectively. 
out-of-roundness profiles (Fig. 14) as obtained after a prolonged etching of the circular (hhl) plates. Arrows now correspond to the $\langle 110\rangle$ direction. On Figure 14 we again depict two different behaviours for the evolution of the profiles with the angle of cut $\theta_{\mathrm{o}}$. A little more rapid evolution is observed for (111) out-of-roundness profiles whereas the shape of out-of-roundness profiles remains quite unchanged for (hhl) cross-sections. In fact the (111) plane constitutes a boundary plane and the more marked changes in shape occur when the (hhl) plane passes through the (111) plane (compare the (112) and (221) outof-roundness profiles for example). Moreover (hhl) out-of-roundness profiles exhibit shapes quite similar to those (Figs. 13d to 13f) obtained for $(\mathrm{hk} 0)$ plates in the vicinity of the (110) plate.
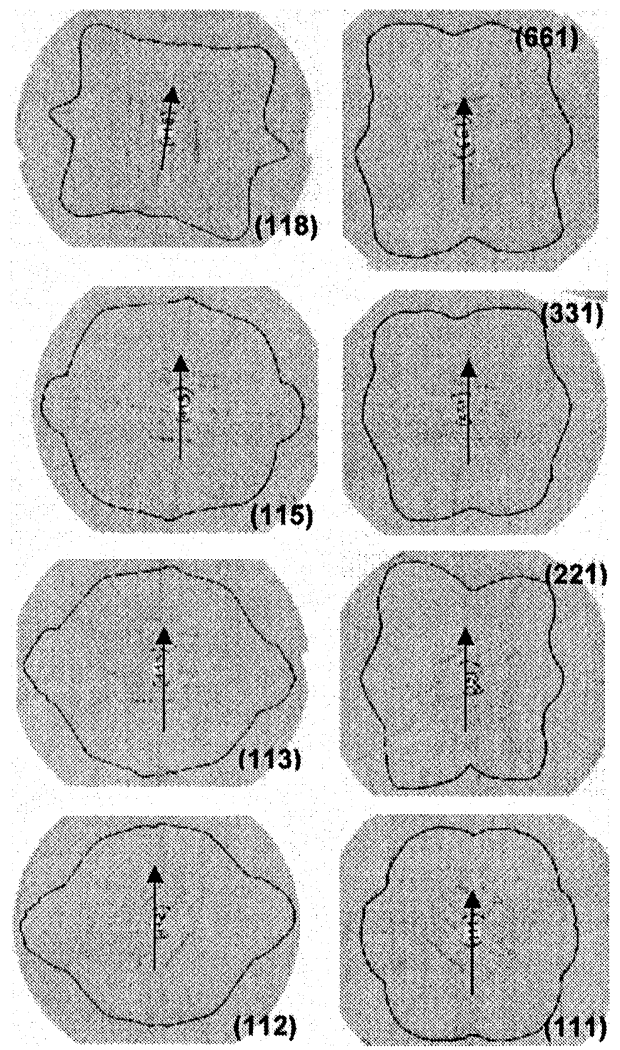

FIGURE 14 Orientation effects in the final out-of-roundness profiles related to various (hhl) sections as indicated on diagrams. 


\section{ANALYSIS OF EXPERIMENTAL RESULTS}

\subsection{Theoretical Basis of the Analysis}

In the following we analyse the experimental etching shapes in terms of the tensorial model for the anisotropic dissolution of crystals [11, 12]. Hence let me recall that in the framework of this model to each surface element ds of orientation $\left(\varphi_{\mathrm{o}}, \theta_{\mathrm{o}}\right)$ we associate a dissolution slowness vector $\mathbf{L}$ whose orientation is that to the unit normal $\mathbf{n}$ to the surface element and whose magnitude $\mathrm{L}\left(\varphi_{\mathrm{o}}, \theta_{\mathrm{o}}\right)$ is equal to the reciprocal of the etch rate $R$. When the angles of cut vary the vector $L$ describes in space a representative surface called the dissolution slowness surface. In the tensorial model the equation of the dissolution slowness surface involves dissolution constants which in fact are the independent coefficients of tensors [11]. The interest of this model lies in the two following properties:

(1) It is possible [16] to calculate the displacement $\mathbf{P}$ of each element ds within the crystal during the dissolution from the equation of the dissolution slowness surface. Thus it is possible to construct numerically 2D and 3D etching shapes from a simulation tool called TENSOSIM [17-19] provided the database (composed of dissolution constants) was determined from experiments.

(2) The tensorial model satisfies the dissolution criteria as stated several years ago by Irving [20]. So if the starting shape is concave the final etching shape must be composed of surface elements corresponding to maxima in the dissolution slowness. Conversely for a starting convex shape minima of $\mathbf{L}$ determine the final etching shape. Note that in the case of a $2 D$ etching shape such as a 2D surface profile or a 2D cross-section of a cylindrical crystal we only consider extrema in the polar diagram of $\mathrm{L}$ lying in the corresponding cross-section [13,19]. But for a 3D etching shape we have to consider extrema associated with peaks and valleys in the dissolution slowness surface $[17,18]$. It should be also pointed out that approximate $2 \mathrm{D}$ etching shapes can be easily deduced from geometrical constructions [3] involving only profile elements associated with extrema in the polar diagram (minima for a convex shape, maxima for a concave shape). 


\subsection{Analysis of $2 D$ Etching Shapes}

For a comprehensive analysis of $2 \mathrm{D}$ etching shapes the dissolution slowness must be known. Fortunately previous works $[12,13,19]$ have shown that an out-of-roundness profile constitutes a crude image of corresponding the cross-sectional polar diagram of $\mathbf{L}$. In particular very small departures are obtained between angular positions $\Psi_{e}$ and $\left(\Psi_{v}, \Psi_{p}\right)$ related respectively to extrema $L_{e}$ in the polar graph of $L$ and to valleys (v) and peaks (p) of the out-of-roundness profile. So we can conveniently use the out-of-roundness profiles of Figures 13 and 14 to analyse the experimental traces. But first of all since out-of-roundness profiles offer images of successive sections of the dissolution slowness surface careful examination of Figures 13 and 14 allow us to drawn some primarily conclusions that agree with remarks made in Section 3.1:

(1) Prolate protuberances are aligned along $\langle 111\rangle$ directions. The influence of these accentuated protuberances extent on large angular sectors that is to say on the ranges $19^{\circ}<\theta_{\mathrm{o}} \leq 0^{\circ}$ for (111) planes and $30^{\circ} \leq \varphi_{\mathrm{o}} \leq 45^{\circ}$ for (hk0) planes.

(2) Minor maxima in the dissolution slowness surface occur for $\{100\}$ planes. Effectively all (hk0) out-of-roundness profiles show peaks in the [001] direction.

(3) If we turn attention to the (110) plane it is difficult to conclude because in the corresponding [110] direction the $\{100\}$ and $\{110\}$ out-of-roundness profiles exhibit respectively peaks and valleys.

(4) Minima which on the (110) profile (Fig. 13f) appear for directions close to $\langle 113\rangle$ directions seems to be associated with $\{113\}$ valleys of the dissolution slowness surface because minima are also observed on out-of-roundness profiles when we move away from the (110) section (see Figs. 13d and 13e).

If we now apply the previous model to the analysis of 2D surface profiles it is clear that we have to consider the cross sectional polar diagrams indicated in Table III and that on these polar diagrams we use only an angular sector $\left(-\alpha_{\max },+\alpha_{\max }\right)$ centred on the dissolution slowness of the reference plate. From Table III it appears that $x_{3}^{\prime}$ and $\mathrm{x}_{1}^{\prime}$ traces must show a progressive evolution as pointed out in Section 3.3. The experimental shapes fulfil this requirement. Now can use the 
TABLE III Identification of cross-sectional polar diagrams of $\mathbf{L}$

\begin{tabular}{ll}
\hline$(h \bar{k} 0)$ planes & \multicolumn{1}{c}{$(h h)$ planes } \\
\hline $\mathrm{x}_{1}^{\prime}$ trace: $(001)$ plane & $\mathrm{x}_{3}^{\prime}$ trace: $(110)$ plane \\
{$[001]$ traces: The same } & $\langle 110\rangle$ traces: specific $\left\{\mathrm{h}^{\prime} \mathrm{h}^{\prime} \mathrm{l}^{\prime}\right\}$ \\
& $\begin{array}{l}(h \bar{k} 0) \text { plane* } \\
\text { planes for }(\mathrm{hhl}) \text { and }(111) \\
\text { planes** }\end{array}$ \\
\hline $\begin{array}{l}\text { * Owing to the symmetry. } \\
\text { ** For example the correspondences }(118) \leftrightarrow(441),(111) \leftrightarrow(112),(114) \leftrightarrow(221) \text { can } \\
\text { be easily established. }\end{array}$ &
\end{tabular}

out-of-roundness profiles of Figures 13 and 14 to discuss the effect of the anisotropic etching on experimental traces. At this point it is convenient to recall that the final shape of a profilometry trace $[12,19]$ depends on the nature and on the number of extrema of $\mathbf{L}$ present in the angular sector $\left(-\alpha_{\max },+\alpha_{\max }\right)$ of the polar diagram. Table IV summarises the most significant results of previous studies.

A comparison of experimental results for $x_{1}^{\prime}$ and $x_{3}^{\prime}$ traces displayed in Figures 9 and 7 and of theoretical predictions of Table IV can be undertaken. It appears that this table furnishes comprehensive explanation for the formation of surface profiles with alternate shape $\left(0^{\circ}<\varphi_{\mathrm{o}} \leq 26^{\circ}\right.$ and $30^{\circ}<\varphi_{\mathrm{o}} \leq 45^{\circ}$ for (hk0) planes and $65^{\circ}<\theta_{\mathrm{o}} \leq 81^{\circ}$, $36^{\circ}<\theta_{\mathrm{o}}<64^{\circ}$ and $6^{\circ}<\theta_{\mathrm{o}}<20^{\circ}$ for (hhl) planes).

We can also justify the observed shapes for [001] and $\langle 110\rangle$ traces (Figs. 10 and 8 respectively) on the basis of theoretical results shown in Table IV. Effectively let us recall that as indicated in Table III expected shapes for [001] traces can be deduced from (hk0) out-of-roundness profiles using an angular sector centred on the dissolution slowness $\mathbf{L}(\mathrm{hk} 0)$ of the reference (hk0) surface. So for [001] traces we work with the sector centred on the direction which is perpendicular to the [001] direction. It is thus possible to draw some conclusions on the expected shapes of [001] traces. These conclusions are listed in Table $\mathrm{V}$ where for convenience the number $\mathrm{N}$ and the type of extrema are also indicated. Examination of the Table $\mathrm{V}$ reveals that the adequation between predicted shapes and theoretical shapes is very good for angles of cut in the range $\left(0^{\circ}-26^{\circ}\right)$. The interpretation is a little more complicated for cuts with $\theta_{\mathrm{o}}>30^{\circ}$. For these cuts it is clear that when the inclination $\alpha$ of the surface element is in the range $\left( \pm 10^{\circ}\right)$ the shape of traces is only determined by the presence of a minimum in the polar diagram which occurs for an orientation corresponding to 


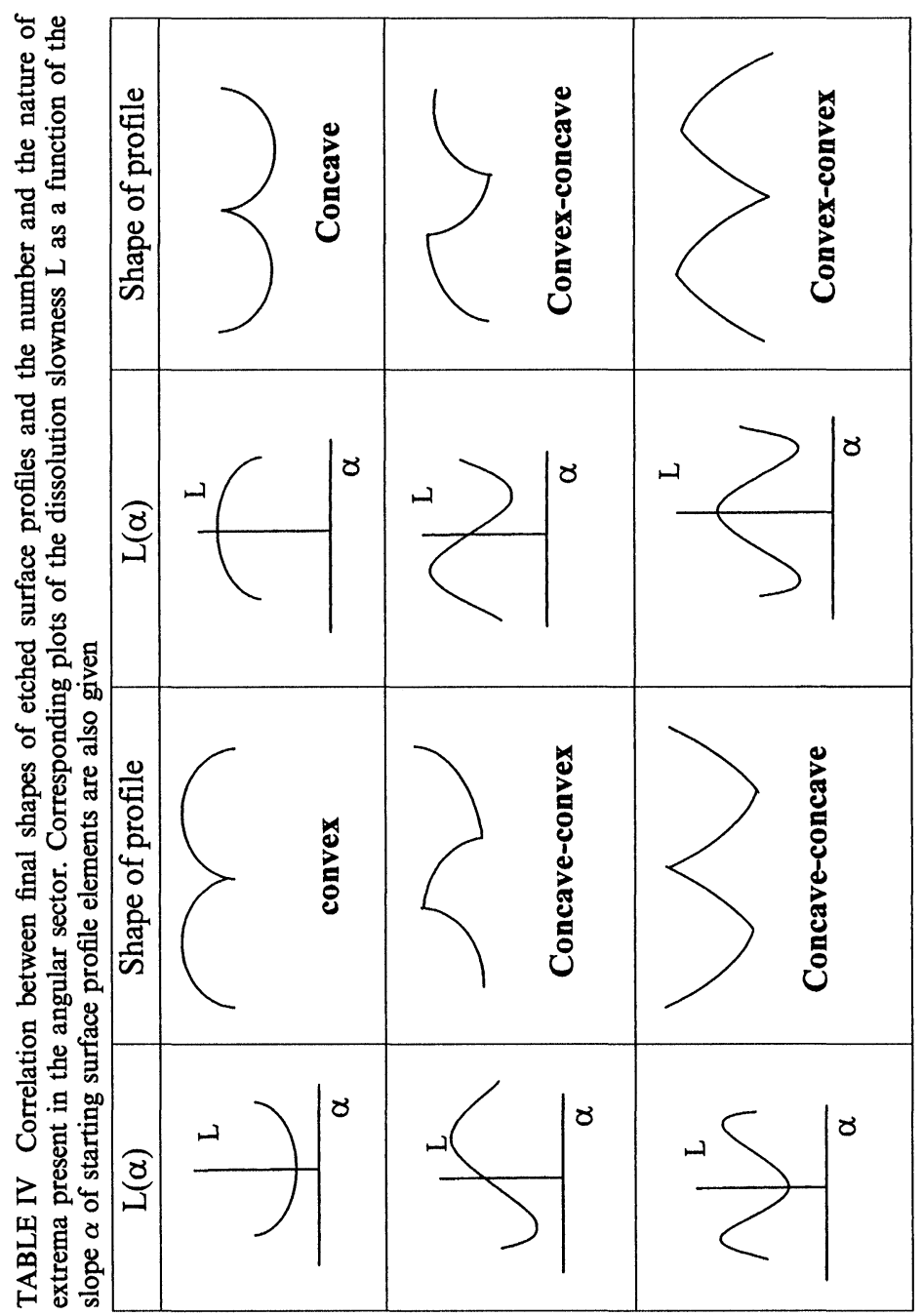




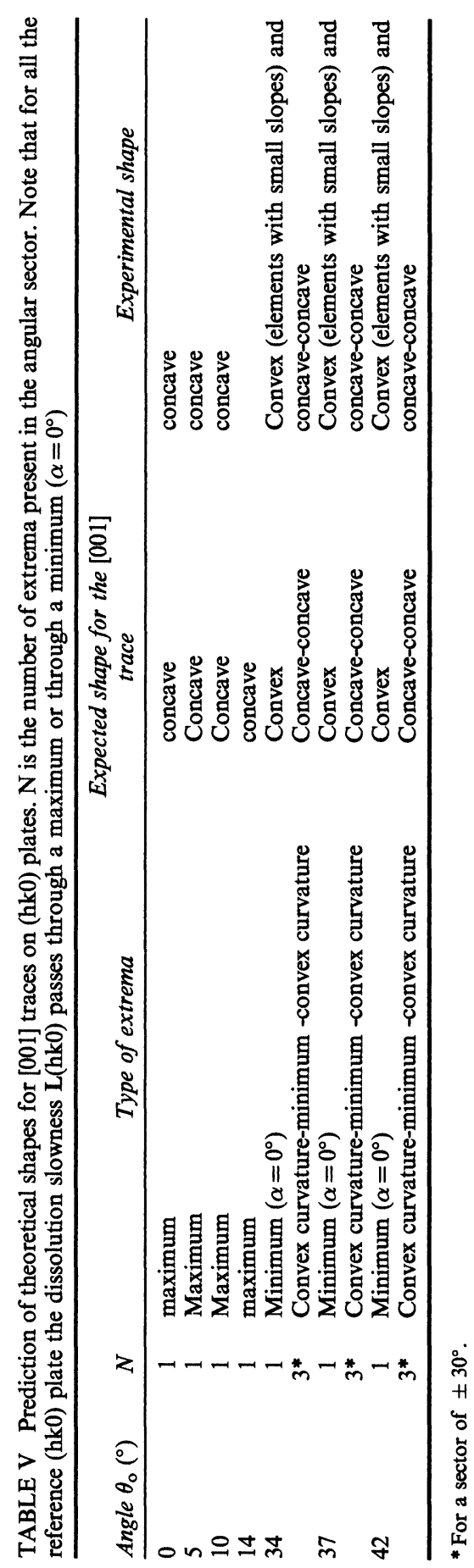




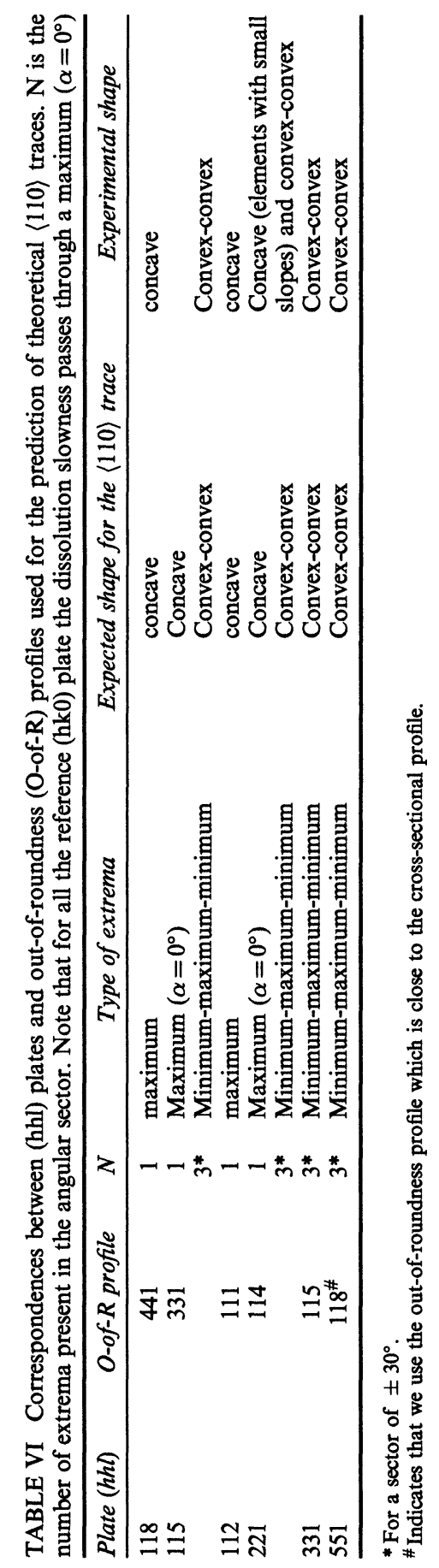


the reference surface $\left(\alpha=0^{\circ}\right)$. But the influence of protuberances associated with $\{111\}$ planes covers a large sector of the dissolution surface. So portions of polar diagram for $|\alpha|>20^{\circ}$ exhibit a convex curvature. The effect of such convex curvatures on the final shape of trace must be certainly close to that due to maxima in L. So on these traces concave curvatures (with large slope) can be observed in accord with experiments.

A similar analysis can be performed for $\langle 110\rangle$ traces made on (hhl) planes taking now into account correspondences between (hhl) planes and cross-sectional planes (see Tab. III). Thus we can establish in Table VI the more probable shape for $\langle 110\rangle$ traces taking attention in this case that the angular sector into consideration is centred on the direction perpendicular to the $\langle 110\rangle$ direction (indicated on the out-ofroundness profiles of Fig. 14). Here again we obtain a good agreement between theoretical predictions and experimental observations.

\section{DISCUSSION AND CONCLUSION}

The chemical etching of silicon crystal in a $35 \% \mathrm{NaOH}$ solution is anisotropic. The anisotropy is characterised by the presence of prolate protuberances in the dissolution slowness surface related to $\{111\}$ minor maxima in the dissolution slowness surface occurs for $\{100\}$ planes. This anisotropy is not far from those observed for other etchants [1-8] even if for the $\mathrm{NaOH}$ solution changes in the dissolution slowness with orientation appear to be different for planes close to the $\{110\}$ planes.

If we turn attention to the degradation of surfaces caused by a prolonged etching we see that as for other etchants $[1,2,4-6,9]$ planes in the vicinity of $\{100\}$ planes exhibit a relatively good smoothness. It is also the case for $\{\mathrm{hhl}\}$ planes close to $\{113\}$ planes. So these orientations for which the $\mathrm{NaOH}$ etching yields surfaces of good quality merit to be considered for the micromachining of new mechanical and optical micro-devices. In the contrary plates close to $\{110\}$ planes which are covered by hillocks appear to be inappropriate for the micromachining of not opened structures such as membranes.

No deviating behaviour results from the comparison of the variations in the etch rate with orientation (Figs. 13d) with the 
out-of-roundness profiles related to (100) and (110) cross-sections. It is in particular the case when we consider the angles of cut for which in the one hand, minima and maxima in the etch rate and in the other hand converse extrema in the polar graph of the dissolution slowness (i.e., in the out-of-roundness profiles) occur. The comparative analysis of etching shapes with predictions deduced from shapes of out-ofroundness profiles show that the tensorial model combined with dissolution criteria can suitably explain final shapes of traces made on (hk0) and (hhl) plates.

At this point of the analysis it is clear that only the micromachining of $3 \mathrm{D}$ structures could provide useful additional information on the anisotropy of the chemical attack of silicon in a $\mathrm{NaOH} 35 \%$ solution. So results on micromachining will be reported in the second part of this paper.

\section{References}

[1] Petersen, K. E. (1982). "Silicon as a mechanical material", Proc. IEEE, 70, 420-457.

[2] Bassous, E. (1978). "Fabrication of novel three-dimensional microstructures by the anisotropic etching of (100) and (110) silicon", IEEE Trans. On Electron Devices, ED-25, 1178-1185.

[3] Sangwal, K. (1987). Etching of Crystals, North-Holland, Amsterdam.

[4] Tellier, C. R. and Brahim-Bounab, A. (1994). "Anisotropic etching of silicon crystals in $\mathrm{KOH}$ solution. Part I: experimental etched shapes and determination of the dissolution slownes surface", J. Mat. Sci., 29, 5953-5971.

[5] Tellier, C. R., Leblois, T. G. and Charbonnieras, A. (2000). "Chemical etching of \{hk0\} silicon plates in EDP Part I: Experiments and comparison with TMAH", Active and Passive Elec. Comp., 23, 37-51.

[6] Charbonnieras, A. R. and Tellier, C. R. (1999). "Characterization of the Anisotropic Chemical Attack of $\{\mathrm{hk} 0\}$ Silicon Plates in a TMAH Solution. Determination of a Database", Sensors and Actuators, 77, 81-97.

[7] Sato, K., Shikida, M., Yamashiro, T., Asaumi, K. and Iriye, Y. (1999). "Anisotropic etching rates of single crystal silicon for TMAH water solution as a function of crystallographic orientation", Sensors and Actuators, 73, $131-137$.

[8] Ju, C. and Hesketh, P. J. (1992). "Measurements of the anisotropic etching of a single-crystal silicon sphere in aqueous cesium hydroxide", Sensors and Actuators $A, 33,191-196$.

[9] Zubel, I. (1998). "Silicon anisotropic etching in alkaline solutions II. On the influence of anisotropy on the smoothness of etched surfaces", Sensors and Actuators $A, 70,260-268$.

[10] IEEE standard on Piezoelectricity, IEEE, New York, 1978.

[11] Brahim-Bounab, A., Amaudrut, J. Y. and Tellier, C. R. (1991). "The dissolution slowness of cubic crystals: Part I. Theoretical and three-dimensional representation for class 23", J. Mater. Sci., 26, 5585-5594. 
[12] Tellier, C. R., Amaudrut, J. Y. and Brahim-Bounab, A. (1991). "The dissolution slowness of cubic crystals: Part II: Applications to class 23 and to combined etching and lithography techniques", J. Mater. Sci., 26, 5595-5607.

[13] Leblois, T. G. and Tellier, C. R. (1992). "Determination of the dissolution slowness surface by study of etched shapes. Morphology of the dissolution slowness surface and theoretical etched shapes", J. Phys. III France, 2, 1259-1286.

[14] Kanda, Y. (1991). "Piezoresistance effect of silicon", Sensors and Actuators A, 28, 83-92.

[15] Durand, S. and Tellier, C. R. (1996). "Linear and non-linear piezoresistance coefficients in cubic semiconductors. I. Theoretical formulations", J. Phys. III France, 6, 237-266.

[16] Tellier, C. R. (1989). "A three-dimensional kinematic model for the dissolution of crystals", J. Cryst. Growth, 96, 450-452.

[17] Tellier, C. R. (1998). "Anisotropic etching of silicon crystals in KOH solution. Part III. Experimental and theoretical shapes for 3D structures micromachined in (hk0) plates", J. Mater. Sci., 33, 117-131.

[18] Tellier, C. R. and Durand, S. (1997). "Micromachining of (hhl) structures: Experiments and 3D simulation of etched shapes", Sensors and Actuators A, 60, $168-175$.

[19] Tellier, C. R. and Brahim-Bounab, A. (1994). "Anisotropic etching of silicon crystals in KOH solution. Part II. Theoretical 2D shapes: discussion of the adequation of the dissolution slowness surface", J. Mater. Sci., 29, 6354-6378.

[20] Irving, B. A., In: The Electrochemistry of semiconductors Edited by Holmes, P. J. Academic press, London, 1962, pp. 262-294. 

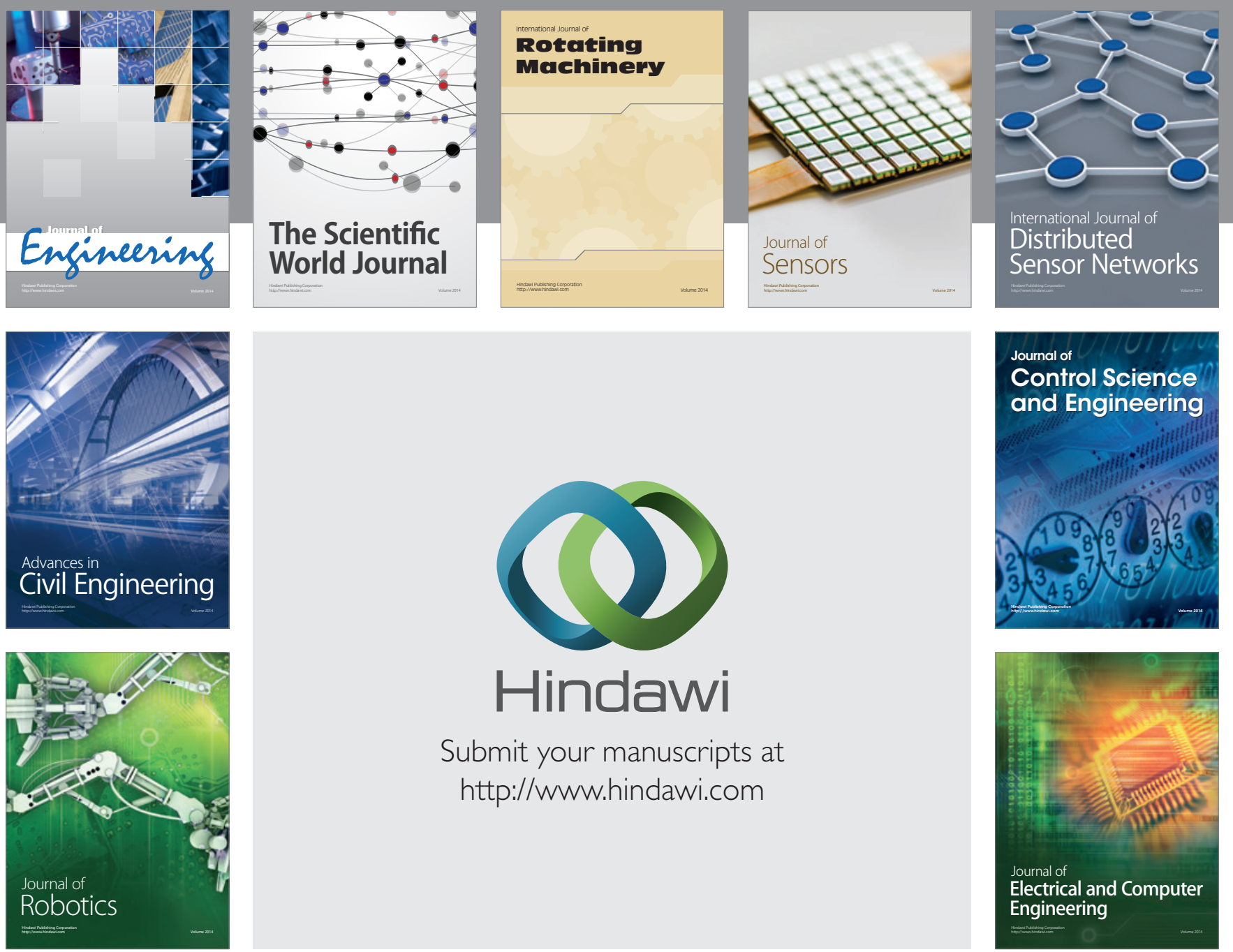

Submit your manuscripts at

http://www.hindawi.com
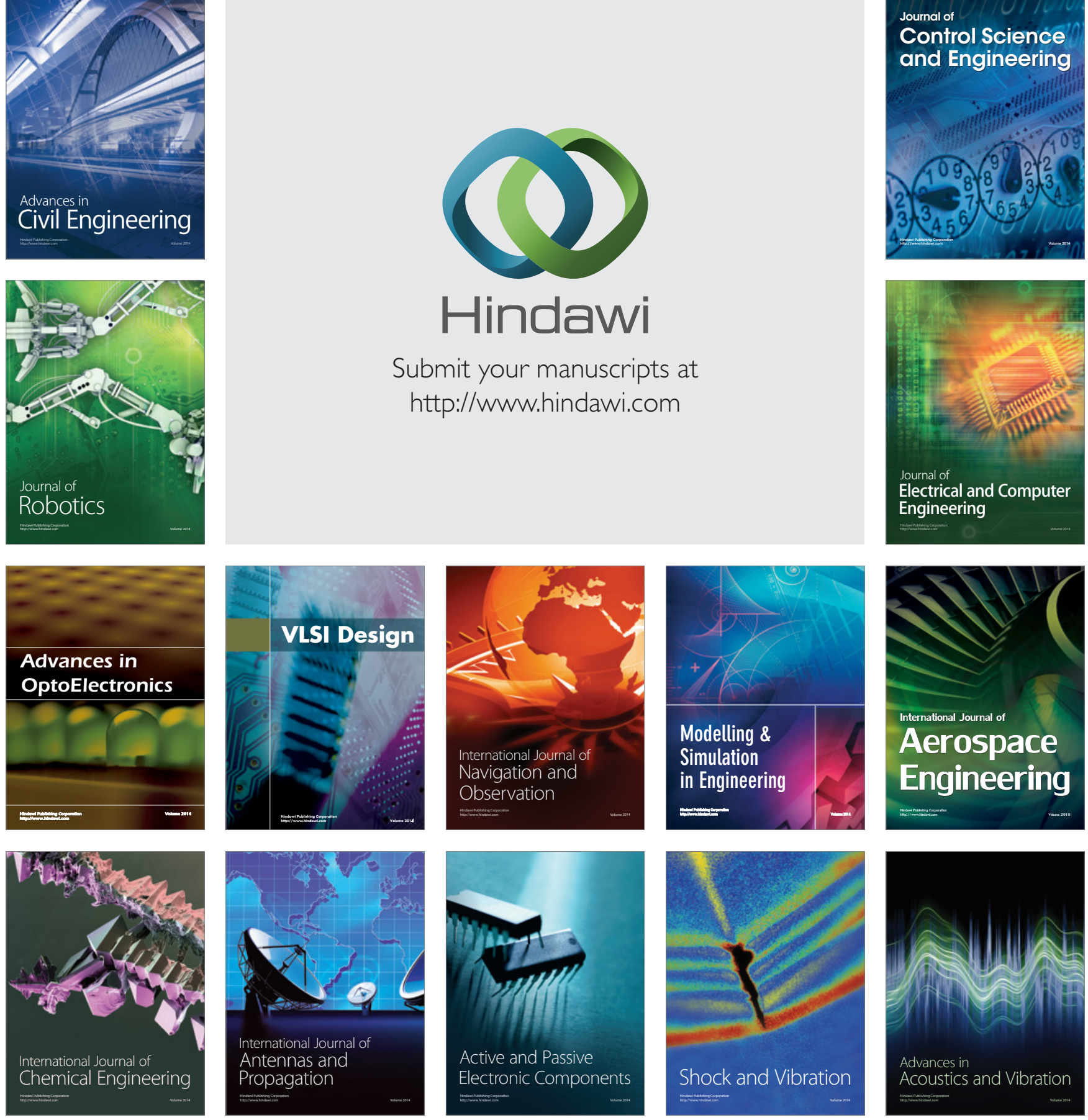